\title{
Exact Map Through Ideal Bends (Again?)
}

\author{
Leo Michelotti \\ Fermi National Accelerator Laboratory \\ P.O. Box 500, Batavia, Illinois 60510
}

June 1995

Presented at the IEEE Particle Accelerator Conference, Dallas, Texas, May 1-5, 1995 


\section{Disclaimer}

This report was prepared as an account of work sponsored by an agency of the United States Government. Neither the United States Government nor any agency thereof, nor any of their employees, makes any warranty, express or implied, or assumes any legal liability or responsibility for the accuracy, completeness, or usefulness of any information, apparatus, product, or process disclosed, or represents that its use would not infringe privately owned rights. Reference herein to any specific commercial product, process, or service by trade name, trademark, manufacturer, or otherwise, does not necessarily constitute or imply its endorsement, recommendation, or favoring by the United States Government or any agency thereof. The views and opinions of authors expressed herein do not necessarily state or reflect those of the United States Government or any agency thereof. 


\title{
Exact map through ideal bends (again?)
}

\author{
Leo Michelotti \\ Fermi National Accelerator Laboratory ${ }^{\dagger}$ \\ P. O. Box 500, Batavia, IL 60510, USA
}

\begin{abstract}
There are three logically independent facets to calculating the transfer map through a bend magnet: physics, geometry, and representation. We will derive the exact map for transit through ideal bends while separating these three, esp., isolating the genmetry problem from the other two.
\end{abstract}

\section{Introduction}

Writing the exact transfer map through ideal bend magnets requires considerations of physics, geometry, and the particular representation in which a particle's state is expressed. Although logically independent, these are frequently mixed together. We will attempt to separate them. It is likely that many people have already gone through this exercise for themselves, but it may be worth repeating.*

\section{Physics}

We all learned the relevant physics as undergraduates. A charged particle in a constant magnetic field, $\vec{B}$, travels at constant speed on a helix aligned along the field. Since the particle experiences no acceleration in the direction of $\vec{B}$

$$
z_{\|}\left(t_{f}\right)=z_{\|}\left(t_{i}\right)+v_{\|} \Delta t
$$

where the subscript " "I" stands in for the appropriate coordinate projection, and $\Delta t=t_{f}-t_{i}$ is the time spent in the magnetic environment. The (radial) frequency of travelling around the helix is,

$$
\omega=|e B / \gamma m|,
$$

in rationalized mks units, where, $e$ is the charge of the particle, $m$ is its mass, and $\gamma=1 / \sqrt{1-\beta^{2}}=E / m c^{2}$ is the usual relativistic factor. Projected onto a plane orthogonal to $\vec{B}$, the helical orbit becomes a circle of radius $\rho$,

$$
\rho=\left|p_{\perp} / e B\right|
$$

where $p_{\perp}$ is the projection of the particle's momentum onto the plane orthogonal to $\vec{B}$.

This is all we need. In the sections to follow we will complete the derivation by (a) solving a few elementary geometry problems and (b) writing the answers in the accelerator physicists' representation.

\footnotetext{
$\dagger$ Operated by the Universities Research Association, Inc, under contract with the U.S. Department of Energy

*The point of doing something this simple is to be reminded that it is simple.
}

\section{Geometry}

The natural Cartesian chart for expressing the geometry, which we will call the " $Z$-chart," has its origin on the helical axis with one coordinate axis parallel to it. Following a standard convention, for bending magnets we choose the $z_{2}$-axis parallel to $\vec{B}$, while for solenoids, we would align $z_{3}$ along the $\vec{B}$, so that in both cases the largest component of momentum is along the $z_{3}$ axis. (Please refure to Figures 1 through 3 repeatedly for visualization of the charts in this paper.) Because it is easiest to work with the transverse equations in terms of spinors, we define complex coordinates $z \equiv z_{3}+i z_{1}$, for bend magnets, or $z \equiv z_{1}+i z_{2}$, for solenoids, and write the transversely projected dynamics in either case as follows. ${ }^{\dagger}$

$$
\begin{aligned}
& z=\rho e^{i \theta}, \quad \theta \in[-\pi, \pi), \\
& \dot{z}=-i \omega z .
\end{aligned}
$$

The time taken to cross the magnetic environment is clearly $\Delta t=-\Delta \theta / \omega$, and we will address below the purely geometric problem of calculating $\Delta \theta$.

A difficulty arises because the orbit is not viewed from the $Z$-chart but from two local charts, say $U_{i}$ and $U_{f}$, which we will call the "initial" and "final" charts, or, alternatively, the "in" and "out" charts. Although these are, in principle, arbitrary, for practical applications, each typically has an axis aligned along $\vec{B}: u_{2}$ for bends, and $u_{3}$ for solenoids. ${ }^{\ddagger}$ In the treatment that follows we will handle only the bend-magnet configuration explicitly, leaving the easier problem of solenoids to the reader.

For bending magnets, then, the $\left(u_{1}, u_{2}\right)$ plane (i.e., $u_{3}=0$ ) is considered the "transverse plane" or "face" of the chart, called so because it is usually considered to be transverse to the local fiducial reference curve, not to the magnetic field. Normally one thinks of the in-face and out-face as located at the edges of the magnet, but this need not be the case; they could be within the body. All that is necessary is that in traversing the region between the faces, the particle is exposed to the environment of a

\footnotetext{
†This definition does have the disadvantage of making $\dot{\theta}$ negative in Eq.(3) and, later, $\Delta \theta=\theta_{f}-\theta_{i}$ negative rather than positive. I've chosen this rather than the less awkward $z \equiv z_{1}+i z_{3}$ for three reasons, not one of which is particularly compelling: (a) it preserves the cyclic ordering of the $\left(z_{1}, z_{2}, z_{3}\right)$ triplet, (b) at least in the U.S., accelerator physicists align the " 3 " axis along the (fiducial) beam current and the "1" axis outward, (c) being at Fermilab, I am accustomed to thinking of beam current as circulating clockwise, and (d) physicists think of time dependence as $e^{-i \omega t}$.

tThis restriction could be removed, but the resulting equations would then have to be solved numerically. In any case, it would not conform to any practical application.
} 


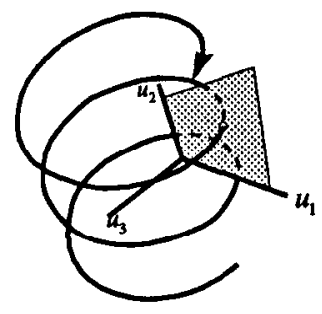

(a)

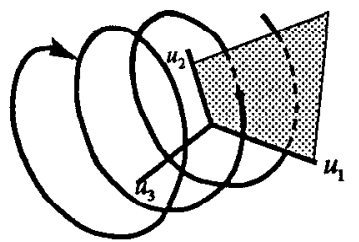

(b)
Figure. 1. Helical orbit in a constant magnetic field, as viewed from a local chart in (a) a bend magnet and (b) a solenoid.

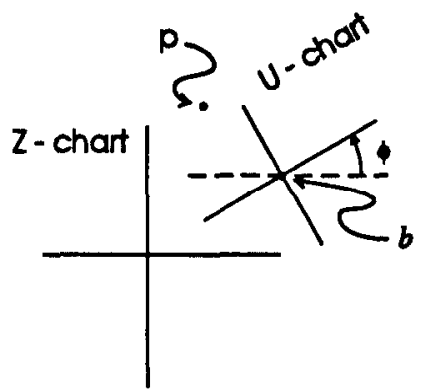

Figure. 2. Complex parameters $b$ and $\phi$.

constant magnetic field. We need to establish chart coordinates for two events, viz., the orbit's intersecting the inand out-faces, the transverse planes of $U_{i}$ and $U_{f}$. The function which converts coordinates of the first event, on $U_{i}$, to those of the second, on $U_{f}$, is the transfer map for the region.

The time $\Delta t$ appearing in $E_{q}$.(1) is nothing but the time interval between these events, Since motion along $\vec{B}$ is taken care of, we can confine our attention to the projection of the orbit on a plane orthogonal to $\vec{B}$ in order to calculate $\Delta t$ and everything else that we need. The geometric problem we must solve requires nothing more complicated than representing circles and lines in a plane. The transformation which takes us from a $U$-chart to the $Z$-chart is written easily in the spinor notation, using $u \equiv u_{3}+i u_{1}$. Referring to Figure 2, we have

$$
\begin{aligned}
& z=b+u e^{i \phi}, \\
& \dot{z}=\dot{u} e^{i \phi},
\end{aligned}
$$

where $b$ is the $Z$-chart spinor coordinate of the $U$-chart's origin, and $\phi$ is the angle of the $u_{3}$ axis relative to the $z_{3}$. Our task now is to represent the very simple motion embodied in Eq.(3) on the $U$ charts and therehy obtain the transfer map $U_{i} \rightarrow U_{f}$. This proceeds in two steps, each of which is a simple geometrical problem: (A) obtaining $b e^{-i \phi}$ given $u$ and $\dot{u}$, and (B) finding the intersection of a line with a circle.

\section{A. What is be $e^{-i \phi}$ ?}

First, using Eq.(4) and Eq.(3), we have

$$
\dot{z}=-i \omega z=-i \omega\left(b+u e^{i \phi}\right) \text {. }
$$

Combining this with Eq.(5) yields

$$
\dot{u}=\dot{z} e^{-i \phi}=-i \omega u-i \omega b e^{-i \phi},
$$

from which we obtain $b e^{-i \phi}$.

$$
b e^{-i \phi}=i \dot{u} / \omega-u
$$

\section{B. Point of intersection between a line and a circle}

We must solve for the point of intersection between the $u_{1}$ axis of a $U$-chart and the orbit projection, which is the circle written in Eq.(2). An arbitrary line $L\left(z_{o}, \eta\right)$ passing through a point $z_{o}$ with direction $\eta$ is the subset,

$$
L\left(z_{o}, \eta\right)=\left\{z_{o}+w e^{i \eta} \mid w \in R\right\}
$$

We easily solve for its intersection with a circle as follows.

$$
\begin{aligned}
z_{o}+w e^{i \eta} & =\rho e^{i \theta} \\
w & =e^{-i \eta}\left(\rho e^{i \theta}-z_{o}\right)
\end{aligned}
$$

Of course, $w$ must be real, so that, by $\operatorname{setting} \operatorname{Im} w=0$, we can find $\theta$.

$$
\sin (\theta-\eta)=\frac{1}{\rho} \operatorname{Im}\left(z_{o} e^{-i \eta}\right)
$$

Applying this to a $U$-chart, we use the line $L(b, \phi+\pi / 2)$; that is, $L$ lies along the imaginary axis of the spinor $u$. In such cases Eq.(7) becomes,

$$
\theta=\phi+\pi / 2-\arcsin \left(\frac{1}{\rho} \operatorname{Re}\left(b e^{-i \phi}\right)\right)
$$

when $\dot{u}$ is attached to $L(b, \phi+\pi / 2)$. We can write this yet another way by applying Eq.(6) to $u$ and $\dot{u}$ evaluated along $L(b, \phi+\pi / 2)$. Thus, $u$ will be pure imaginary, so that

$$
\theta=\phi+\pi / 2+\arcsin (\operatorname{Im}(\dot{u}) /|\dot{u}|) .
$$

\section{Of magnets and maps}

We are now ready to complete the construction of a transfer map. Of course, on the $Z$-chart the "initial" and "final" spinor coordinates are trivially related by a phase rotation.

$$
z_{f}=z_{i} e^{i \Delta \theta}, \quad \dot{z}_{f}=\dot{z}_{i} e^{i \Delta \theta} .
$$

Eq.(4) and Eq.(5) can now be used to obtain $u_{f}$ and $\dot{u}_{f}$.

$$
\begin{aligned}
\dot{u}_{f}= & \dot{u}_{i} e^{i(\Delta \theta-\Delta \phi)} \\
u_{f}= & u_{i} e^{i(\Delta \theta-\Delta \phi)}+\left(b e^{-i \phi}\right)_{i} e^{i(\Delta \theta-\Delta \phi)} \\
& -\left(b e^{-i \phi}\right)_{f}
\end{aligned}
$$

The complete transfer map, $\left(u_{i}, \dot{u}_{i}\right) \mapsto\left(u_{f}, \dot{u}_{f}\right)$ is now constructed according to the following procedure.

\section{Bend algorithm}

Step 1. Use Eq.(6) to evaluate $\left(b e^{-i \phi}\right)_{i}$.

$$
\left(b e^{-i \phi}\right)_{i}=i \dot{u}_{i} / \omega-u_{i} .
$$


Step 2. Given $\left(b e^{-i \phi}\right)_{i}$, from Step 1 , construct $\left(b e^{-i \phi}\right)_{f}$ using the relative in-face to out-face geometry. We will illustrate below how to do this.

Step 3. Calculate $\Delta \theta-\Delta \phi$ by applying Eq.(8) to both faces.

$$
\begin{aligned}
\Delta \theta-\Delta \phi= & \arcsin \left(\operatorname{Re}\left(b e^{-i \phi}\right)_{i} / \rho\right) \\
& -\arcsin \left(\operatorname{Re}\left(b e^{-i \phi}\right)_{f} / \rho\right)
\end{aligned}
$$

Step 4. Finally, use Eqs.(10) and (11) to complete the map. Notice that $\Delta \theta-\Delta \phi$ appears only in the argument of an exponent. Rather than use Eq.(12) directly we can employ

$$
e^{i(\Delta \theta-\Delta \phi)}=\frac{\sqrt{1-\left(\operatorname{Re}\left(b e^{-i \phi}\right)_{i} / \rho\right)^{2}}+i \operatorname{Re}\left(b e^{-i \phi}\right)_{i} / \rho}{\sqrt{1-\left(\operatorname{Re}\left(b e^{-i \phi}\right)_{f} / \rho\right)^{2}}+i \operatorname{Re}\left(b e^{-i \phi}\right)_{f} / \rho} .
$$

\section{A. Rectangular bends}

Detailed information about the magnet's geometry, embodied in the relative placements of the in- and out-faces, is used in Step 2. The simplest possibility is the rectangular bend. We take the "in" and "out" faces to be parallel, so that $\phi_{i}=\phi_{f} \equiv \phi$. From the obvious relation, $b_{f}=b_{i}+L e^{i \phi}$, where $L$ is the length of the magnet, we obtain the result,

$$
\left(b e^{-i \phi}\right)_{f}-\left(b e^{-i \phi}\right)_{i}=L
$$

which is to be fed directly into Step 3 .

\section{B. Sector bends}

Sector bends are only a little more complicated but easily handled by representing, on the $Z$ chart, the point of intersection between the imaginary axes of the $U_{i}$ and $U_{f}$ charts. To position these, we place the face of each orthogonal to a local, fiducial path that would be followed by an "ideal" particle. Let $\bar{\rho}$ symbolize its radius of curvature. Further, placing the origins of the $U$-charts on the fiducial path the intersection point has coordinate $-i \bar{\rho}$ on each chart. Thus, using Eq.(4), we have,

$$
\left(b e^{-i \phi}\right)_{f}-\left(b e^{-i \phi}\right)_{i}=\left(1-e^{-i \Delta \phi}\right)\left(i \bar{\rho}-\left(b e^{-i \phi}\right)_{i}\right) \text {. }
$$

We note in passing the usual relation between $\Delta \phi, \bar{\rho}$, and magnet length, $L$, recalling that, by our convention, $\Delta \phi<0$,

$$
e^{-i \Delta \phi}=\left(\sqrt{1-(L / 2 \bar{\rho})^{2}}+i L / 2 \bar{\rho}\right)^{2} .
$$

Keeping $L$ fixed and letting $\vec{\rho} \rightarrow \infty$ then reproduces Eq.(13).

\section{Arbitrarily angled faces}

The in- and out- faces can be rotated through additional angles. If we use the MAD convention for specifying angles $c_{1}$ and $c_{2}$, effectively we must replace $\phi_{i} \rightarrow \phi_{i}+e_{1}$ and $\phi_{f} \rightarrow \phi_{f}-e_{2}$ in Eq.(14). The result then looks as follows.

$$
\left(b e^{-i \phi}\right)_{f}=\left(b e^{-i \phi}\right)_{i} e^{-i \Delta \phi}+i \bar{\rho}\left(e^{-i e_{2}}-e^{i e_{1}} e^{-i \Delta \phi}\right)
$$

Setting $e_{1}=-e_{2}=\Delta \phi / 2$ and using Eq.(15) again reproduces Eq.(13).

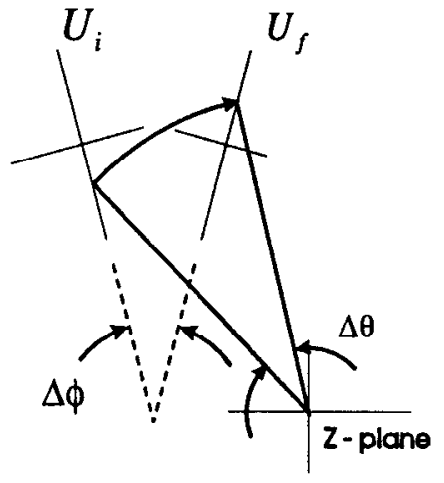

Figure. 3. Relationships between the $U_{i}, U_{f}$, and $Z$ charts.

\section{Representation}

The final complication arises when we insert the accelerator physicists' coordinate representation into these expressions. A particle's crossing the plane of a "face" is typically recorded using momentum and energy coordinates normalized by the fiducial. The position sector is recorded by transverse coordinates $x_{1}, x_{2}$, and a time offset, $c \Delta \tau$, while the momentum sector is represented by normalized transverse components $s^{\S}$ of $\vec{q} \equiv \vec{p} / \bar{p}$, and a total momentum offset, and $\delta \equiv|\bar{p}| / \bar{p}-1$. To apply the bend algorithm, we need only write $\vec{v}$ in terms of these and then use the spinor $\dot{u}=v_{3}+i v_{1}$.

Starting from,

$$
\vec{\beta}=\vec{v} / c=\vec{p} c / E=\vec{q} \cdot \vec{p} c / E
$$

we substitute

$$
E^{2}=\vec{p}^{2} c^{2}+m^{2} c^{4}=(\bar{p} c)^{2}(1+\delta)^{2}+m^{2} c^{4},
$$

to obtain

$$
\vec{\beta}=\vec{q} / \sqrt{(1+\delta)^{2}+(m c / \bar{p})^{2}} .
$$

We can obtain $v_{1}$ and $v_{2}$ from this directly, but since $q_{3}$ is not recorded, we must get $v_{3}$ by using the following.

$$
\begin{aligned}
E^{2} & =\vec{p}^{2} c^{2}+m^{2} c^{4}=\vec{p}_{\perp}^{2} c^{2}+p_{3}^{2} c^{2}+m^{2} c^{4} \\
\frac{E^{2}-m^{2} c^{4}}{\bar{p}^{2} c^{2}} & =\left(\vec{q}_{\perp}\right)^{2}+\left(q_{3}\right)^{2} \\
\beta_{3} & =q_{3} / \sqrt{\cdots} \\
& =\sqrt{\frac{(1+\delta)^{2}-\left(\vec{q}_{\perp}\right)^{2}}{(1+\delta)^{2}+(m c / \bar{p})^{2}}}
\end{aligned}
$$

Of course, there's even more fascinating material to wade through, but we're already up against the three page limit and have no more space (see edge of page below). $\Lambda$ finished version will follow somewhere, sometime, but not here, and not now.

\$That is, the projections into the in- and out-faces. 